\title{
Investigation of Feature Extraction Methods for Image Retrieval Application
}

\author{
${ }^{1}$ BusettiSaiHarsha ${ }^{2}$ Shamruthaa T R ${ }^{3} \mathrm{Hema}$ Pa ${ }^{4}$ Priscilla ShaminR ${ }^{5}$ Thusnavis Bella Mary I \\ ${ }^{1,2,3,4}$ Student, ${ }^{5}$ Asst. Professor, Karunya Institute of Technology and Sciences, Coimbatore, India \\ Email: ${ }^{1}$ busettisaiharsha@karunya.edu.in, ${ }^{2}$ shamruthaatr@karunya.edu.in, ${ }^{3}$ hemapa@karunya.edu.in, \\ ${ }^{4}$ priscillashaminr@karunya.edu.in, ${ }^{5}$ bellamary@karunya.edu
}

\begin{abstract}
Content-based image retrieval is a technique used for retrieval of desired images via their colour, texture, and shape features. Features play a major role in an image. The major challenge in image retrieval lies in extracting the optimal features from an image. Feature extraction is a process of selecting optimal low level feature subsets. It transforms the input image into a set of features that describes the image with sufficient accuracy. In this paper, three specialized features i.e. colour moments, Region properties and Grey Level Co-Occurrence Matrix (GLCM) are extracted. This Image retrieval system using the hybrid features are tested using Corel image datasets consisting of 1000 images from 10 semantic categories. The efficiency of the system is evaluated in terms of precision, recall and error rate. From the experimental results, we can conclude that these hybrid features have improved the precision of the retrieval system when compared with other state-of-the-art methods.
\end{abstract}

Keywords: Content-based image retrieval- Feature extraction. Colour moments. GLCM- Region Properties

\section{INTRODUCTION}

The advancement of digital technology has remarkably increased the digital images which are used in countless of applications, such as medicine,agriculture, journalism, ilitary, crime investigation, and so on. There is a need for efficiently retrieving the image from the digital image database. As different methods and techniques use images for many purposes, it is important to retrieve the exact image for good performance metrics.

The CBIR also known as query image content (QBIC) utilizes low level features in order to represent the index of the image. Progressive changes are continually made by the research community for obtaining effective results.

Generally, low-level and high-level visual features exist. In this paper, we are working on basic low-level features.

We propose an efficient and effective CBIR system by using low level features. To identify color information of an image, colour moments in HSI colour space is used and for texture, we use GLCM features, and for shape features we use geometric properties.

Color is widely used low level feature in CBIR. To identify colour feature there are color histogram [3], color moments [2], Gabor filter[6], and color autocorrelation[1] under a certain color space. In this paper we are using a color moment which measures the characteristic color distribution in an image and uniquely describes a probability distribution. The main use of colour feature is for color indexing purpose which compares the similarity of two images based on color. This process is achieved by comparing the given query image to a database of Corel images in order to find and retrieve similarity images.

Texture feature is another low level feature used to indicate quality of the image in addition to colour features as they are not sufficient to identify the image because different images may have similar histogram. Texture features give us more details about specific region to an image. There are many texture analysis method such as GLCM/GLSD[6],Gabor Wavelet[1-2], Wavelet moment[3],Quantized Histogram[4],Local Binary Pattern[7],Binarized Statistical Image Features(BSIF)[2].Among these we are using Grey-Level Co-occurrence Matrix(GLCM) represents the statistical method for the spatial relationship of pixels also known as the grey-level spatial dependence matrix.

For describing shape features we have Wavelet transforms[1],Canny edge extraction[7], Hue moment shape feature[6], Sobel operator[5].Here we are using region based properties for representing shapes which specifies the objects body within the closed boundary.

The rest of the paper is organised as follows: division IIdefines the methodology of the work, division IIIdefines the results and the last division gives theconclusion and future work. 


\section{METHODOLOGY}

To improve the efficiency of the retrieved image, the procedureas displayed in figure 1 is followed.

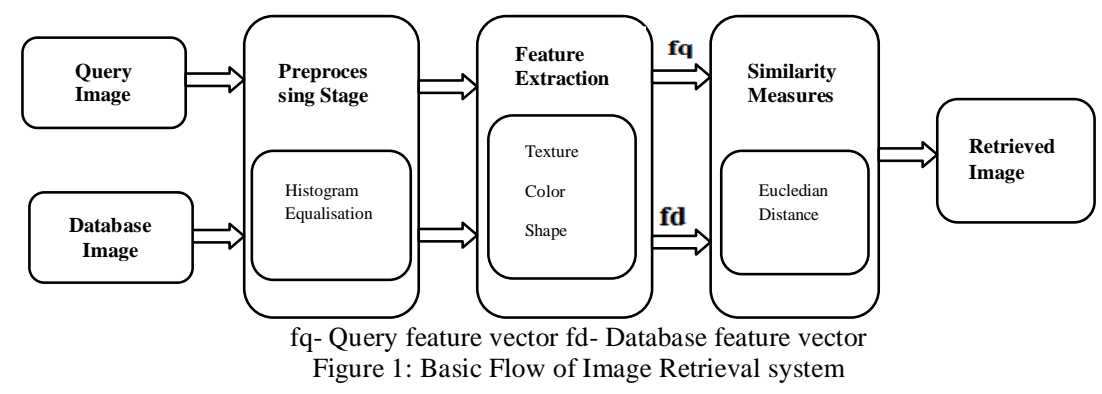

A. Input database: The input database contains images from Corel database $(1 \mathrm{~K})$. From this database 10 images are taken as query images and 990 images are taken as database images.

B. Pre-processing:It is the initial stage in Image retrieval system. It enhances some image features or quashes undesired distortion for further processing. Histogram equalization adjusts image intensities to enhance contrast which is achieved by persuasively spreading out the most intermittently intensity values.

C. Feature extraction:Feature extraction makes the image proficient for scrutiny and categorizing. In feature extraction certain features of interest in an image is further processed. We have considered the common lineaments like shape, texture and colour. Texture feature extraction:According to the prospect specification to retrieve an meticulous image many types of texture feature extraction methods are used.In our work we are going for GLCM which is a analytical method.

Shape feature extraction:

Generally shape is described as the outer edge of the image.For any object shape is a basic feature to portray its content. For efficient shape feature extraction Region Properties are used.They are

1) Area:

2) Eccentricity

3) Euler number

4) Convex area

5) Perimeter

7) Orientation

Colour feature extraction:

Colour feature is widely used visual feature. Colour moments technique is experimented to extract colourfeatures. Two steps are used for this purpose:

(1)In first step, input RGB image is separated into

$\mathrm{R}, \mathrm{G}$ and $\mathrm{B}$ component images.

(2) Second step involves computation of mean (which describes the centre of probability distribution of the signal) and standard deviation of each of the component. Colour moments generates 6 -D feature vector.

\section{III.EXPERIMENTAL RESULTS}

\section{A. Similarity Measurements}

Similarity measurement is another important issue in CBIR in which the input query image is compared with other database images for the similarity by using Euclidean distance.

$$
D_{E}=\sqrt{ } \sum_{i=1}^{n}\left(\left|I_{i}-D_{i}\right|\right)^{2}
$$

\section{B. Performance Metrics}

The retrieval performance of the present method is measured efficiently in terms of Precision(P) and Recall(R).We can define them as follows

$$
\begin{gathered}
P=\frac{N r}{I} \\
R=\frac{N r}{N t}
\end{gathered}
$$

Wherelis the number of images retrieved that is similar to the query image, $N_{r}$ is the count of relevant images from retrieved images, and $N_{t}$ is the total countof relevant images available in the database. If the precision is closer to 1 it indicates the system is more effective.

\section{RESULTS}

The proposed method tests the hybrid features (colour, texture, and shape features). This database is implemented in Matlab2013b. For the query image, top 10 matches are retrieved. P@10 is calculated.

To validate the results comparison graph is drawn in Figure 6. The proposed method is compared with the method in [31]. The average precision obtained here is $78 \%$. Comparison is 
International Journal of Innovative Research in Applied Sciences and Engineering (IJIRASE)

Volume 1, Issue 12, DOI: 10.29027/IJIRASE.v1.i12.2018.228-232, June 2018

done for all categories in the dataset. For all the categories the implemented method achieved higher average precision than the approach used in Method [31].

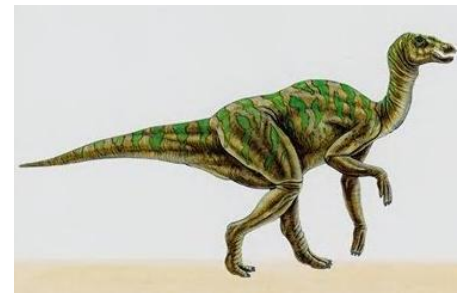

Figure 2: Input Query Image

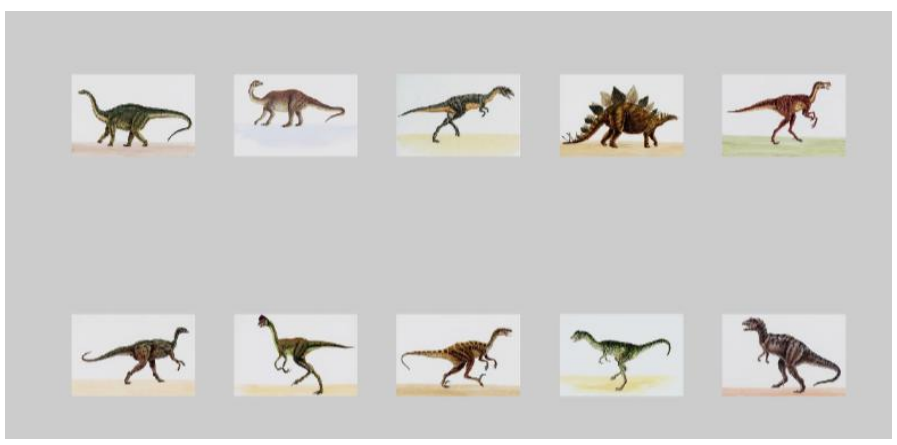

Figure 3: Experimental Results For Dinosaur

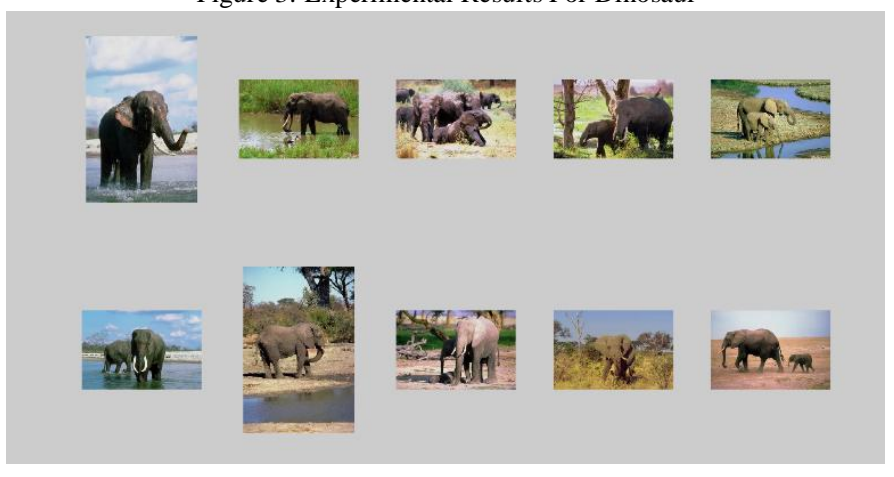

Figure 4: Experimental Results For Elephant

Table 1

Average Precision and Recall

\begin{tabular}{|c|c|c|}
\hline Category & $\begin{array}{c}\text { Precision } \\
(I=10)\end{array}$ & Recall $(I=10)$ \\
\hline Bus & 1 & 0.1 \\
\hline Elephant & 1 & 0.1 \\
\hline Horses & 0.9 & 0.09 \\
\hline African & 0.8 & 0.08 \\
\hline Mountains & 0.5 & 0.05 \\
\hline Beaches & 0.6 & 0.06 \\
\hline Food & 0.5 & 0.05 \\
\hline Dinosaur & 1 & 0.1 \\
\hline Buildings & 0.7 & 0.07 \\
\hline Roses & 0.8 & 0.08 \\
\hline
\end{tabular}


International Journal of Innovative Research in Applied Sciences and Engineering (IJIRASE)

Volume 1, Issue 12, DOI: 10.29027/IJIRASE.v1.i12.2018.228-232, June 2018

\begin{tabular}{|l|l|l|}
\hline Average & $\mathbf{0 . 7 8}$ & $\mathbf{0 . 0 7 8}$ \\
\hline
\end{tabular}

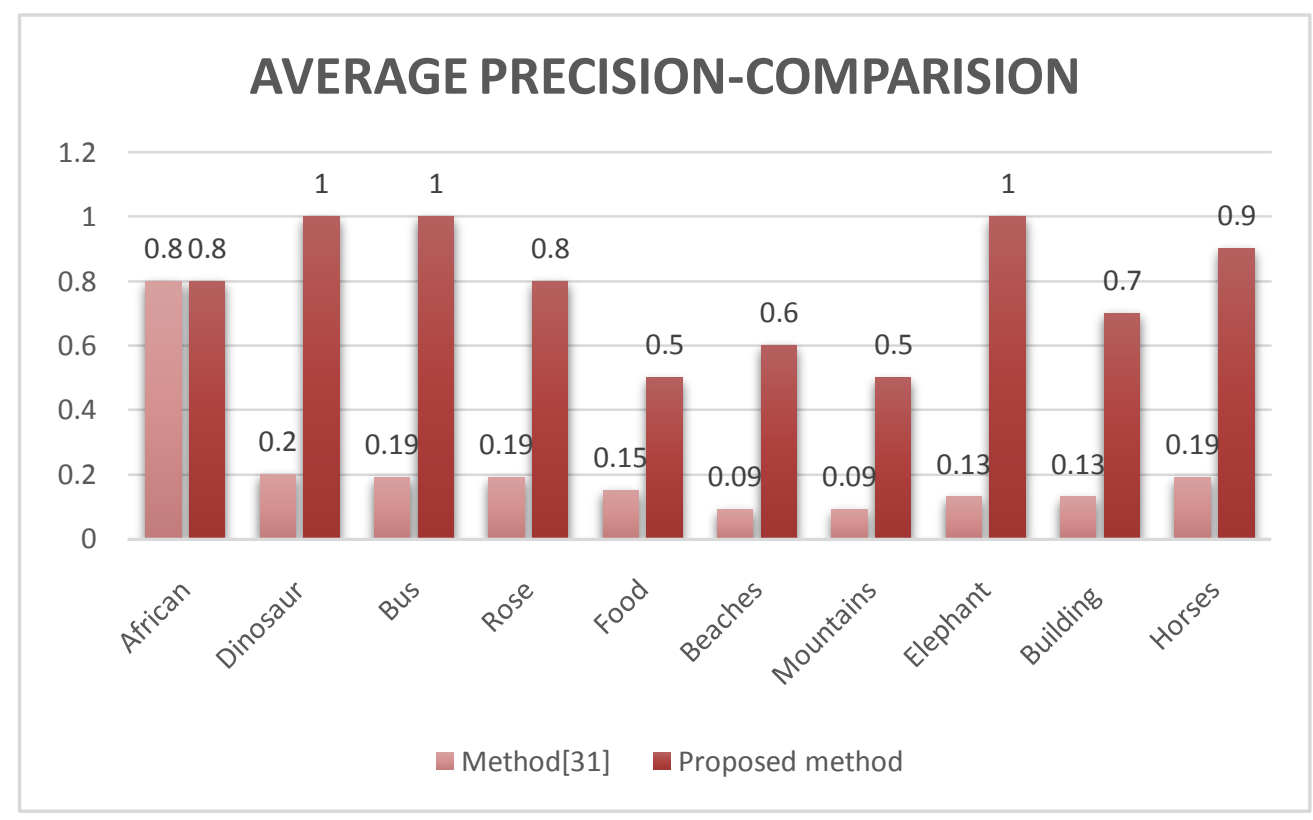

Figure 6: Comparison of Proposed approach with Method from [31]

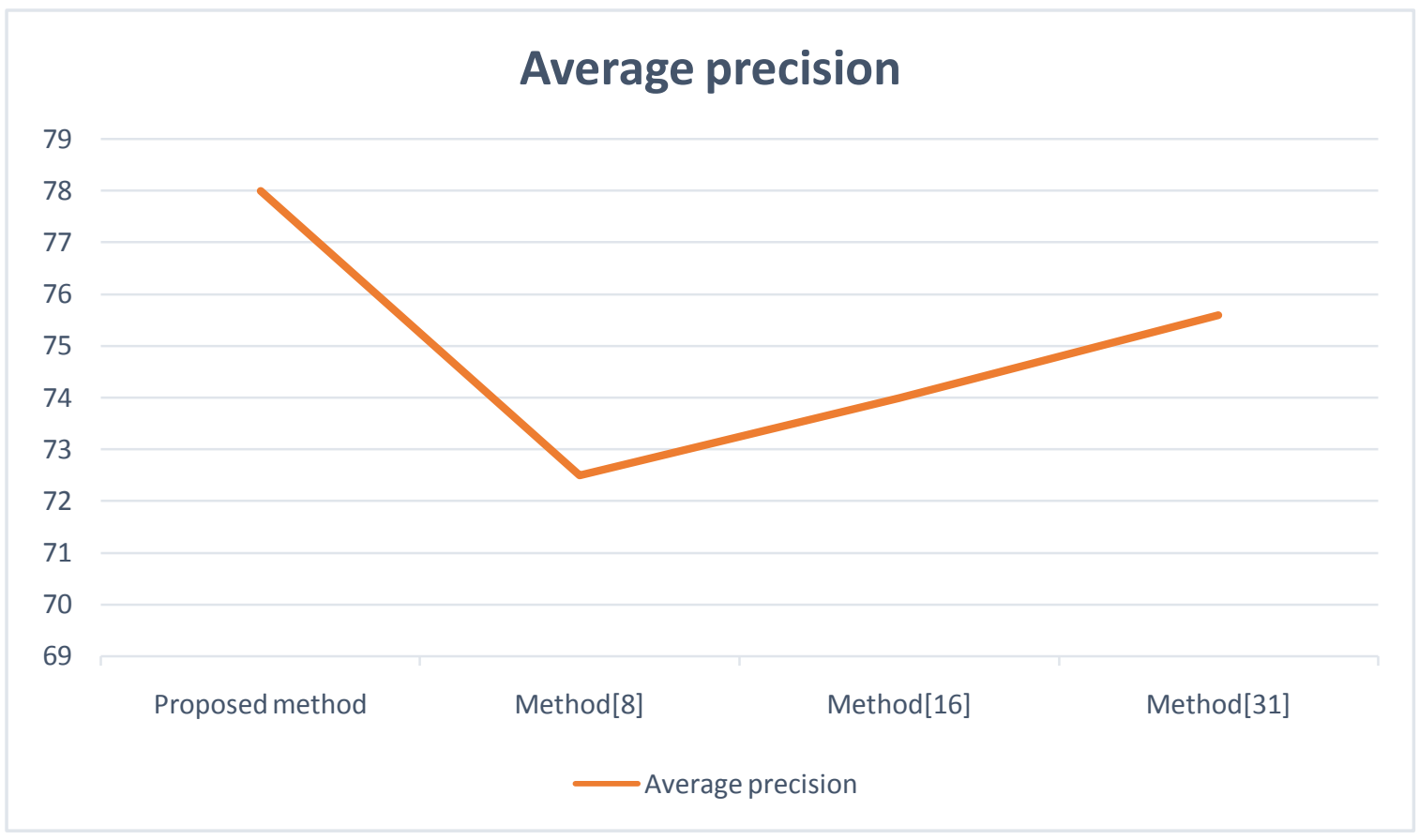

Figure 7: Comparison of proposed method with other methods.

\section{V.CONCLUSION AND FUTURE WORK}

This paper adopted GLCM, region properties and color moments features for integrating the relations between textures, shape and color. The proposed technique performance is tested on coral database. For similarity measurement Euclidean distance is a used. The experimental result indicates that the suggested model gained a percentage of $78 \%$ in terms of precision when related with other models.For future work the performance of the implemented system can be combined with other special features. 


\section{REFERENCES}

[1] A.Anandh, Dr.S.Suganya, Dr.K.Mala"Content Based Image Retrieval System based on Semantic Information Using Color, Texture and Shape Features"

[2]YogitaMistry, D.T. Ingole, M.D. Ingole "Content based image retrieval using hybrid features and various distance metric"

[3]ZeyadSafaaYounus, Dzulkifli Mohammad, TanzilaSaba "Content based image retrieval using PSO and K-means clustering algorithm"

(Geosciences,Springer, 2014)

[4]FazalMalik ,BaharumBaharudin

"Analysis of distance metrics in content-based image retrieval using statistical quantized histogram texture features in the DCT domain"

[5] Ming Zhang,Ke Zhang, Qinghefeng, Jianzhong Wang, Jun Kong, Yinghua Lu

"A novel image retrieval method based on hybrid information descriptors"

[6] PranotiP.Nane and NarendraG.Bawane

"An effective technique for the Content Based Image Retrieval to reduce semantic gap based on an optimal classifier technique" [7]L.K.Pavithra, T.SreeSharmila "An efficient framework for image retrieval using color, texture and edge features"

(Elsevier-2017)

[8] M.Subrahmanyam, Q.M.Jonathan Wu, R.P.Maheshwari, R.Blasubramanian, " Modifiedcolor motif co-occurrence matrix for image indexing and retrieval" Computers and Electrical engineering, Elsevier, 39 (2013), pp. 762-774

[9] I.Jeena Jacob, K.G.Srinivasagan, K.Jayapriya, “ Local Oppugnantcolor texture pattern for image retrieval system".Pattern Recognition letters, Elsevier, 42 (2014)pp.7278.

[10] Jing Yu, Zengchang Qin, Tao Wan, Xi Zhang. " Feature integration analysis of bag-of-features model for image retrieval" Neurocomputing, Elsevier, 120(2013)PP.355-364

[11] Anil BalajiGonde, R.P. Maheshwari,R.Balasubramanian, “ Modified Curvelet transform with vocabulary tree for content based image retrieval", Digital signal Processing, Elsevier, 23(2013) pp. 142-150

[12] Santosh Kumar Vipparthi, S.K Nagar, "Expert image retrieval system using directional local motif XoRpatterns",Expert systems with applications, Elsevier, 41(2014), pp. 8016-8026.

[13] Guang -Hai Liu, jing-Yu Yang, " Content-based image retrieval using color difference histogram", Pattern recognition, Pattern Recognition, Elsevier, 46(2013), pp.188-198.

[14] Alex Papushoy,AdrianG.Bors," Image retrieval Based on query by saliency content",Digital signal processing, Elsevier, 36(2015),pp.156-173.

[15] ElaYildizer, Ali MetinBalci, Mohammad Hassan, RedaAlhajj," Efficient content-based image retrieval using multiple support vector machines ensemble" Expert systems with applications, Elsevier 39(2012), pp.2385-2396.

[16] M.Subrahmanyam, R.P.Maheshwari, R.Balasubramanian,"Expert system design using wavelet and color vocabulary trees for image retrieval" Expert systems with applications, Elsevier 39(2012),pp.5104-5114.

[17] SubrahmanyamMurala, R.P Maheshwari, R.Balasubramanian," Local Tetra patterns: A new feature descriptor for content based image retrieval", IEEE Transactions on Image processing, Vol 21, No.5, 2012.

[18] Huayuan, Xiao-Ping Zhang, "Statistical modelling in the wavelet domain for compact feature extraction and similarity measure of images" IEEE Transactions on circuits and systems for video technology, Vol.20, No.3, 2010.

[19] Robert m.Haralick, K.Shanmugam, Dinstein,'Textural features for image classification" IEEE Transactions on systems, Man and Cybernetics, Vol.3, No.6, 1973.

[20] Arnold W.M Smeulders, Marcel Worring, Simone santini, Amarnath Gupta, Ramesh Jain," Content-based image retrieval at the end of the early years", IEEE transactions on pattern analysis and machine intelligence, Vol.22, No.12, 2000.

[21] Chuen-horng Lin, Rong-Tai Chen, Yung-kuanchan, "A smart content based image retrieval system based on color and texture feature" Image and Vision computing, Elsevier 27(2009), 658-665

[22] Daniel Cerra, MihaiDatcu,"A fast compression-based similarity measure with applications to content based image retrieval" J. Vis. Commun. Image R. Elsevier 23(2012) pp.293302.

[23] Ahmed Talib, MassudiMahmuddin, HusnizaHusni, LoayE.George, "A weighted dominant color descriptor for content based image retrieval" . Vis. Commun. Image R. Elsevier 24(2013)pp.345-360.

[24] M.EmreCelebi, Hassan A.Kingravi, Alp Aslandogan, " Non linear vector filtering for impulsive noise removal from color images" Journal of Electronic Imaging,IS\&T, SPIE, 16(3): $033008,2007$.

[25] C.Kenney, Y.Deng, B.S. Manjunath, G.Hewer, “ Peer Group Image enchancement" IEEE Transactions on image processing, Vol.10, No.2, February 2001.

[26] Michael S.Lew, NicuSebe, ChabaneDjeraba, Ramesh Jain," Content based multimedia information retrieval", ACM Transactions on multimedia computing, Communications and Applications, vol. 2, No.1, 2006.

[27]Min huang, HuazhongShu, Yaqiong ma, Qiuping gong, "Content based image retrieval technology using multi-feature fusion" Elsevier Optik 126(2015), pp. 2144-2148.

[28] NishantShrivastava, VipinTyagi, "An efficient technique for retrieval of color images in large databases" Elsevier Computers and Electrical engineering 46(2015), pp. 314-327.

[29] Muhammad Imran, RathiahHashim, Noor Elaiza, "Content based image retrieval using color layout descriptor and generic fourier descriptor" Lecture notes in Electrical Engineering 315, Springer 2015

[30] Meng Zhao, Huaxiang Zhang, jiande Sun,” A novel Image retrieval method based on multi-trend structure descriptor" J.Vis. Commun.Image R. 30(2016), pp.73-81.

[31]Amandeep Khokher ${ }^{1}$, Ranjeesh Talwar ${ }^{2}$

"A fast and effective Image retrieval scheme using color-texture and shape based histogram."

(2017-Multimedia tools) 